\section{Yes, but am I free?}

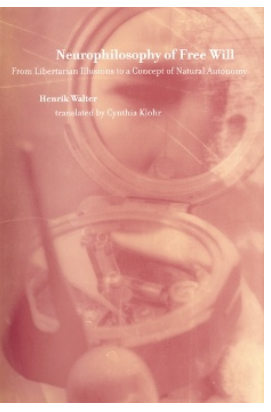

\section{Neurophilosophy of Free Will}

\author{
by Henrik Walter, translated by Cynthia Klohr \\ MIT Press, Cambridge, Massachusetts, 2001. \$45 \\ hardcover, pp 391 \\ ISBN 0-262-23214-6
}

Reviewed by Adina L. Roskies

Walter's ambitious book, translated from German, asks the perennial question "Do we have free will?" The book is a combination of conceptual analysis and scientific survey, with emphasis placed on the brain sciences. Walter's approach differs from other approaches to the question of free will. Some consider the link between free will and moral responsibility to be central, and argue for the presence (or absence) of free will from evidence that we are (or are not) morally responsible. Others think that the answer to the free will question depends upon whether or not our complete physics is deterministic. In contrast, Walter grants that we may well have a deterministic physics, but argues that within this framework, there may still be room to carve a place for free will, both by reformulating our conception of what free will requires, and by careful attention to our brains and how they give rise to intention and action. As he puts it, "the task of the neurophilosopher is to discover which interpretation of free will has the greatest empirical plausibility." The uniqueness of his approach lies not so much in his naturalism, but in his focus upon the brain, rather than physical law in general, as the key to determining whether or not we are free.

Walter argues that the libertarian concept of free will-that we have free will because the world is indeterministic - is incorrect. Here the ground is well-trodden: libertarians are traditionally attacked on two fronts. On the one hand, the claim that physics is indeterministic is questionable, and, as Walter notes, there is little evidence to support the contention that quantum indeterminacy

Adina Roskies is in the Department of

Linguistics \& Philosophy, Massachusetts

Institute of Technology, 77 Massachusetts

Avenue, Building E39-245, Cambridge,

Massachusetts 02139, USA.

e-mail:adinad@mit.edu is significant in brain function. On the other hand, and more interestingly, if our decisions or actions are not determined, they are random, and freedom of the will seems incompatible with randomness. As a consequence, compatibilist philosophers have argued that some form of determinism must hold in order for us to lay claim to our actions. Walter goes further, attempting to construct a philosophical theory in which freedom and determinism are compatible on a sound neuroscientific base.

Walter identifies three components of an action done freely: first, the agent could have done otherwise; second, the agent acts for intelligible reasons; third, the agent is the originator of his own actions. He then examines each in turn, and considers how we might interpret these three criteria in a neurophilosophical context. Consider the first component, for example. In a deterministic universe, there is no possibility of doing otherwise in a given situation. Walter supposes that doing otherwise in a similar situation may suffice for free will. He then suggests that chaos theory might provide a model for how we can understand doing otherwise: in chaotic systems, small differences in initial conditions can result in wildly different behavior. Walter likewise brings other philosophical and neuroscientific theories to bear upon the second and third components: he invokes naturalistic theories of semantics when discussing the issue of the intelligibility of actions, and uses theories and data from the neuroscience of emotion in developing and clarifying a concept of authenticity, which is the self-identification and appropriation of one's actions. The last third of the book is devoted to explaining these concepts and the theories and data involved in their formulation and support. He draws widely from neuroscientific evidence, citing data as disparate as EEG recordings and lesion studies, and drawing from established as well as speculative the- ories. Walter ultimately concludes that we have what he calls "natural autonomy," a type of free will that differs from that suggested by our ordinary intuitions.

While unfailingly interesting, the book has noticeable shortcomings. Walter's relatively uncritical approach to the neuroscientific literature and to how it relates to his arguments is troublesome. For instance, he surveys the literature claiming that the brain is chaotic, and concludes rather prematurely that there is ample evidence that it is. Whether or not this is the case, it is insufficient for his purposes that neurophysiological data with a signature reminiscent of chaotic systems exists; for chaos to be involved in the free will debate, the right kind of brain system must be shown to be chaotic. Walter presents no evidence, for example, that the specific neural systems involved in decision-making are chaotic, or even seem to be so. Nor is there a need to invoke chaos theory at all if all we require to undergird freedom is the ability to act differently in a similar situation. Ordinary nonchaotic deterministic systems can do that. Moreover, Walter's undisciplined style is itself problematic: although his writing is lively and generally good, in his enthusiasm to convey the richness and breadth of the philosophical and scientific theories that underlie and play into the free will debate, he tries to do too much, sketching sometimes tangential and sometimes central positions with such broad strokes that he fails to adequately cover often technically difficult and complex material. I would have liked to see him a little more judicious in his choice of what to include in this dense book, and more deliberate in presenting the details and commitments of the positions he does include. Finally, there are the glaring failures in (one hopes) translation or editing that result in a few neologisms, not infrequent misuse of words, and numerous typographical errors. In the best cases, these errors are merely annoying or even amusing, but in the worst they obscure the exposition by drastically altering the meaning of a sentence or by rendering technical formulas nonsensical.

Whereas Neurophilosophy of Free Will provides a good coarse-grained view of the intellectual landscape in which debates about free will, determinism and neurophilosophy reside, it falls short as either an introduction or an insider's guide to the area. With respect to the intellectual terrain at issue, the book is more like a satellite image than a road map: In his attempt to survey all the relevant issues, Walter's own argument is obscured. In the end, the reader finds herself without a clear idea of where she has arrived, let alone whether she is free. 